\title{
Departamento de Productos Forestales y Conservación de Bosques del Instituto de Ecología, A.C.
}

\author{
Michael R. Keyes Hennin ${ }^{1}$ \\ Raymundo Dávalos Sotelo ${ }^{1}$
}

\section{LOS PRODUCTOS FORESTALES EN LOS CAMBIOS GLOBALES}

\section{Antecedentes e introducción}

El aprovechamiento de los recursos forestales y en particular, de su parte más importante desde el punto de vista comercial, la madera, constituye un aporte significativo al Producto Interno Bruto de muchas naciones y estos recursos forestales son, por lo tanto. El objeto de estudio de un gran número de centros de investigación de esos países. En el estudio de estos recursos intervienen especialistas de diversas disciplinas, desde la ingeniería forestal hata la biología, pasando por arquitectura, ingeniería civil, ecología, diseño industrial, etc., sin dejar de tomar en cuenta desde luego, la relativamente reciente carrera de ingeniero de tecnología de la madera. A manera de ejemplo de la contribución de algunas de estas especialidades, podemos mencionar que las ingenierías química y mecánica son aplicadas en la transformación de los extractivos y fibras de plantas para surtir las necesidades de la sociedad.

En su forma tradicional, los productos del bosque básicamente no han sido comercializados en su conjunto. No obstante, materias primas como: fibras para amarre, leña, carbón, plantas medicinales, ornamentales, frutos silvestres hojas y elementos para la construcción vernácula aún se encuentran entre los satisfactores más importantes del bosque en el medio rural. Resinas, celulosas, y materiales para la fabricación de muebles y estructuras han contribuido a la base de la industria occidental como productos forestales en la era moderna.

La ingeniería se a aplicado en diversos campos, desde la maquinaria en la extracción, procesamiento de productos primarios como madera aserrada, chapa y madera contrachapada, tableros aglomerados, pulpa, madera reconstituida etc. Hasta en la reglamentación y optimación y uso de insumos para la producción de diversos artículos para bienestar humano. Todo ello partiendo de la base de que la investigación sobre los productos forestales es para el beneficio tanto de los dueños de los bosques como de los industriales, quienes dependen de los bosques como fuente de materia prima. El uso eficiente de los recursos naturales, se supone, podría traducirse en una industria competitiva que protegería su base de materia prima en forma sostenida.

Las necesidades nacionales han absorbido los esfuerzos de los pocos investigadores en productos forestales en México. El desconocimiento de las especies leñosas de México y sus usos más adecuados, son algunos de los retos para los grupos de investigación de la República.

1 Investigadores. Departamento de Productos Forestales y Conservación de Bosques. Instituto de Ecología, A.C. Apdo. Postal 63. Xalapa, Ver., 91000. México. 
Partiendo de que el árbol se encuentra en manos de los industriales, el Laboratorio de Ciencia y Tecnología de la Madera, ha generado diversas investigaciones a nivel teórico y práctico. Algunos de sus éxitos sobresalientes, se han dado en el área de ingeniería estructural, con la producción de las Noras Técnicas Complementarias para Diseño y Construcción de Estructuras de Madera (DDF, 1987b) y sus Comentarios (Robles, et. al, 1988), (Fig.1), que forman

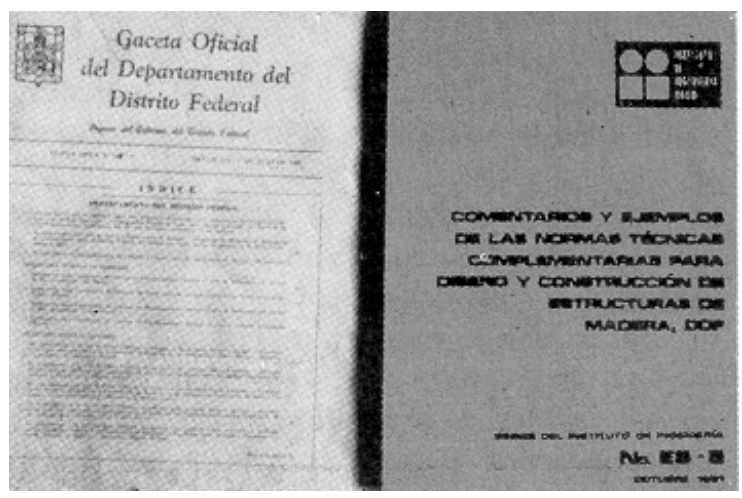

Figura 1. Normas Técnicas y Comentarios parte el Reglamento de Construcciones del Distrito Federal (DDF, 1987a); los estudios de las maderas de la selva Lacandona (Bárcenas, et. al, 1980: Bárcenas, 1994) y los que sirvieron de base para la elaboración de las normas de dimensiones y de clasificación para madera de pino (Dávalos, et. al., 1984); Echenique-Manrique, et. al., 1987) Ordóñez y Dávalos, 1985; DGN, 1983; DGN, 1985)

En fechas más recientes, la elaboración de un manual de Diseño por parte de personal de LACITEMA exclusivamente (Ricalde, 1988) (Fig.2) y, la participación de la redacción de un Manual de Construcción de Estructuras Ligeras bajo los auspicios de la Comisión Forestal de América del Norte significativos al uso racional de la madera en estructuras. Otras áreas de influencia son:

1. La elaboración de normas mexicanas sobre diversos temas: preservación (DGN, 1983), protección de estructuras contra termitas (DGN, 1983), especificaciones de comportamiento para tableros (DGN, 1994), etc.

2. Diseño de productos a partir de madera mobiliario escolar, estufas de secado solar (Martínez-Pinillos, 1984) (Fig.3); muebles de oficina, salas, comedores, camas, etc., todos

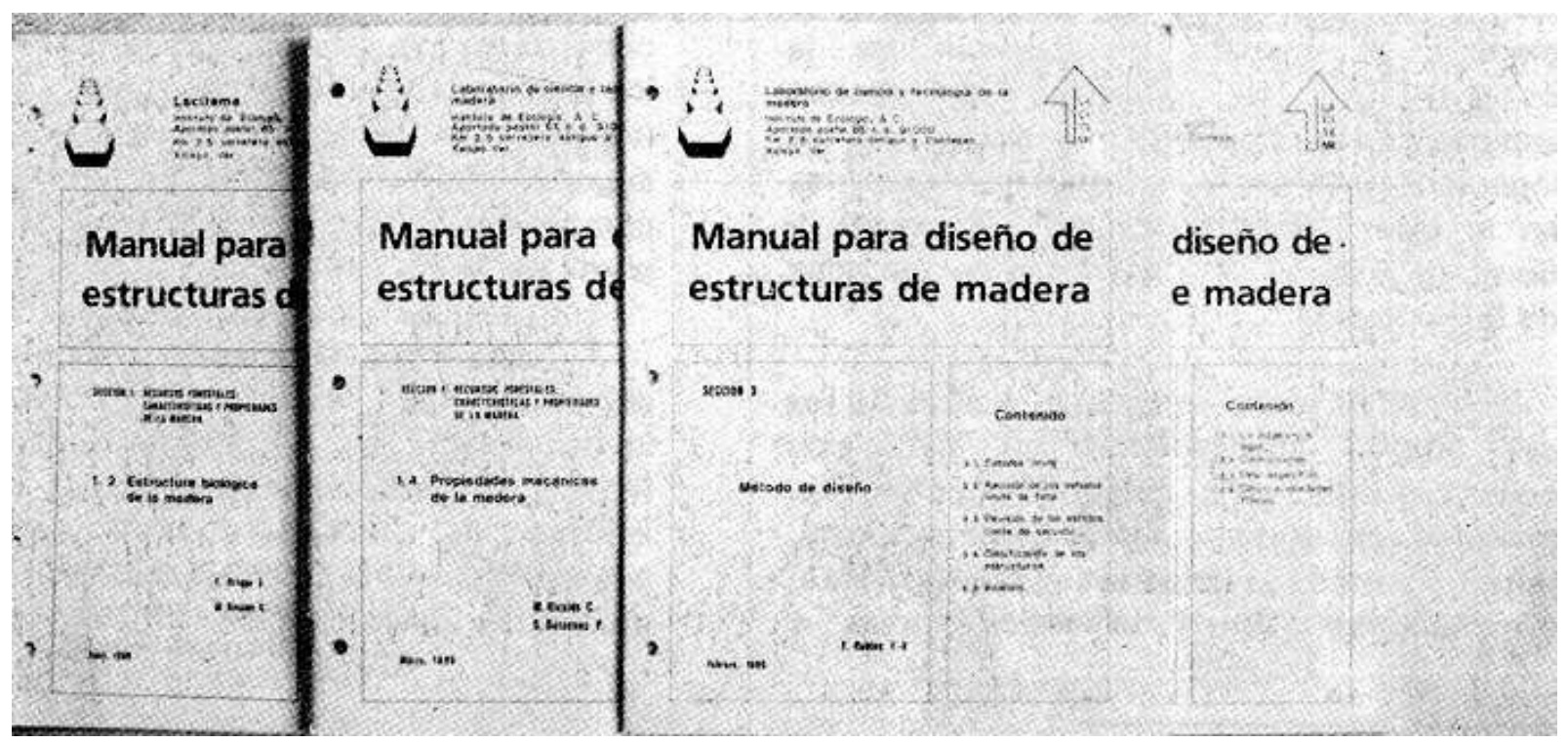

Figura 2. manual para Diseño de Estructuras de Madera 
ellos con madera sólida (LACITEMA s.f.). Los experimentos con madera doblada (Martinez-Pinillos, 1990), también han dado resultados exitosos, como lo destaca el hecho de haber obtenido en 1985 un premio nacional por el diseño de una silla-sillón.

Todo ello sin olvidar, los estudios básicos sobre tecnología de la madera anatomía, incluyendo la xiloteca "Faustino Miranda" (Ortega et. al. 1988) (fig.4); preservación (fig5); biodeterioro (Pérez, et al, 1977), y propiedades físicas y mecánicas (Dávalos, et al, 1977; Ordóñez, et al, 1989).

En el campo de la difusión y la información, cabe resaltar la creación desde 1980 de una Unidad de Información especializada en madera, la que en su fase inicial fue planeada por INFOTEC y alimentada con parte del acervo particular del Dr. Ramón Echenique y con libros y documentos de la biblioteca del desaparecido INIREB.
Posteriormente, el CONACYT apoyó de manera significativa la adquisición de libros. A la fecha, en su cmpo es una de las más importantes en el país( Evans y Arizmendi, 1991); en la difusión de la ciencia, las revistas "La madera y su uso en la construcción" y "Nota técnica" y posteriormente" La madera y su uso", han sido casi únicas en su género (Fig.6); ya que muy poca de la información que generan otras instituciones es publicada; por lo que es difícil encontrar datos sobre los usos de las especies mexicanas. El Boletín LACITEMA (Ruelas, 1993), editado desde 1980, ha servido como vínculo entre el Laboratorio y los tecnólogos de México.

Finalmente, es importante resaltar, que con el apoyo de las autoridades del Instituto de Ecología, A.C., del CONACYT y los integrantes de LACITEMA, se ha logrado mantener en operación e incrementar el acervo de la Unidad de Información.

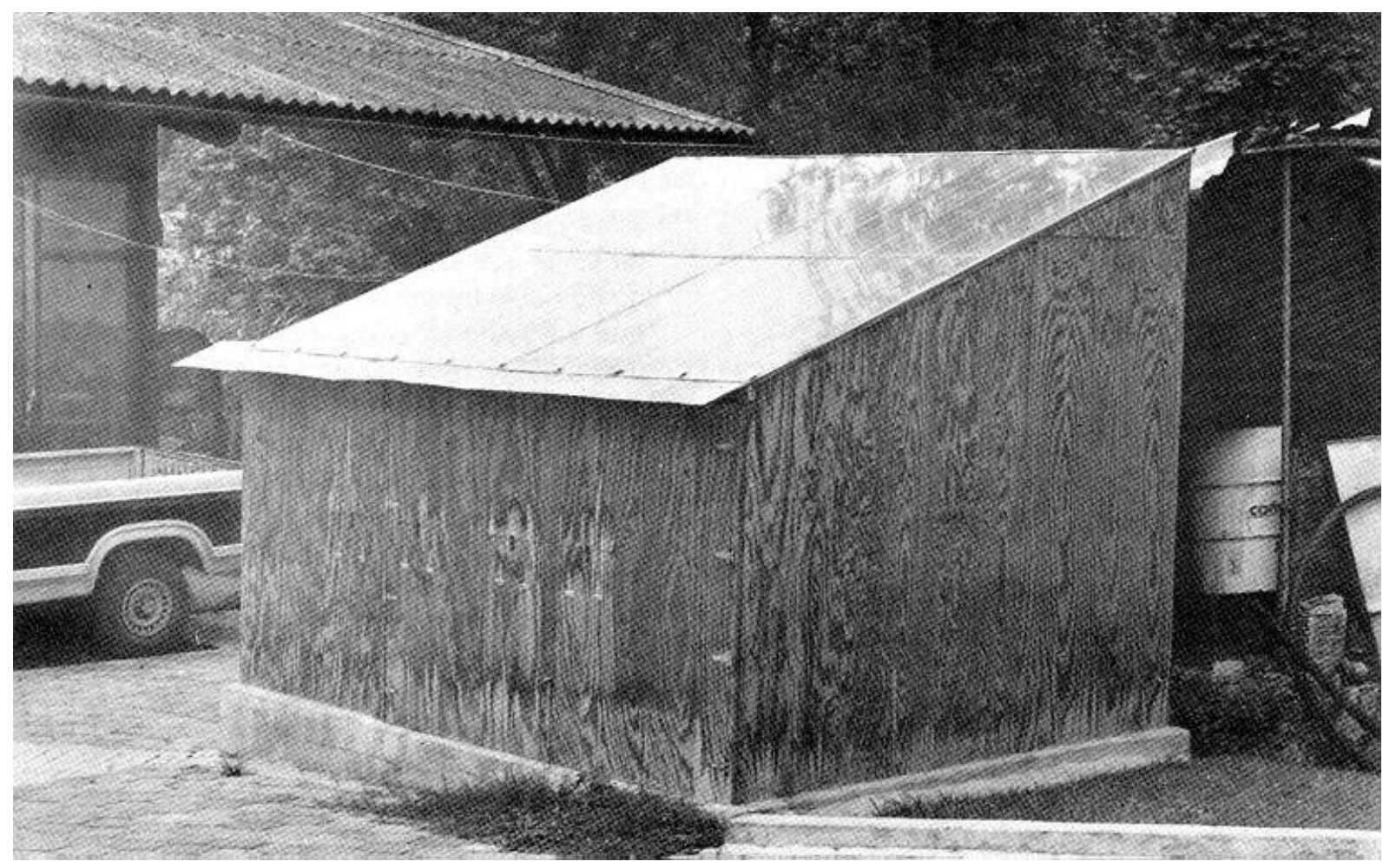

Figura 3. Estufa de Secado Solar 


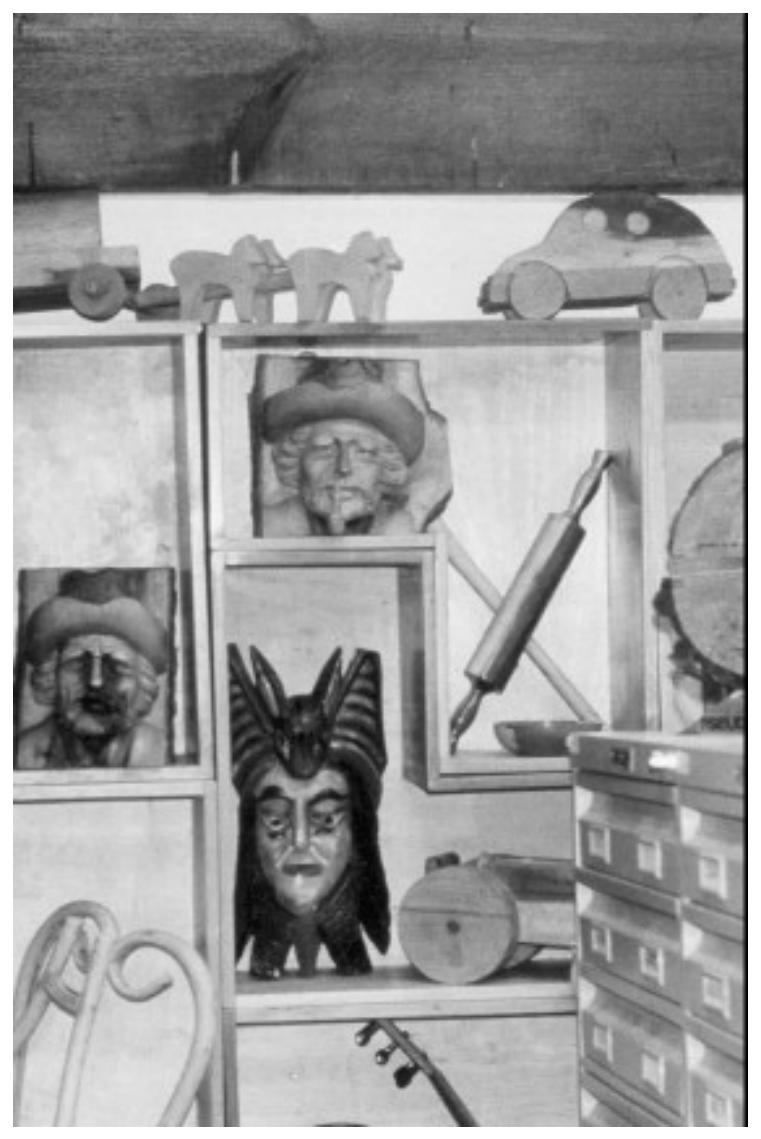

Figura 4. Xiloteca "Faustino Miranda"

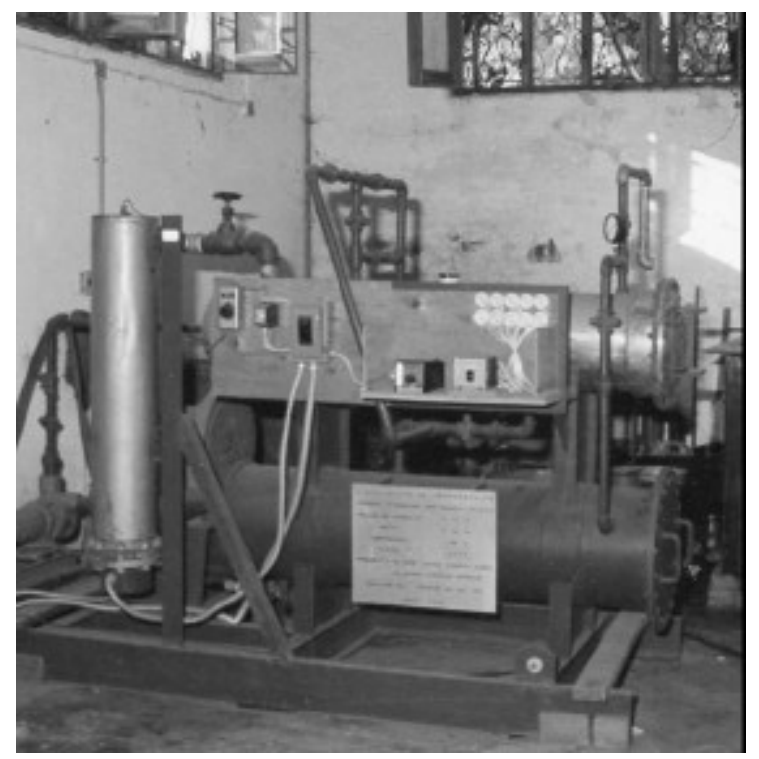

Figura 5. Planta de Impregnación

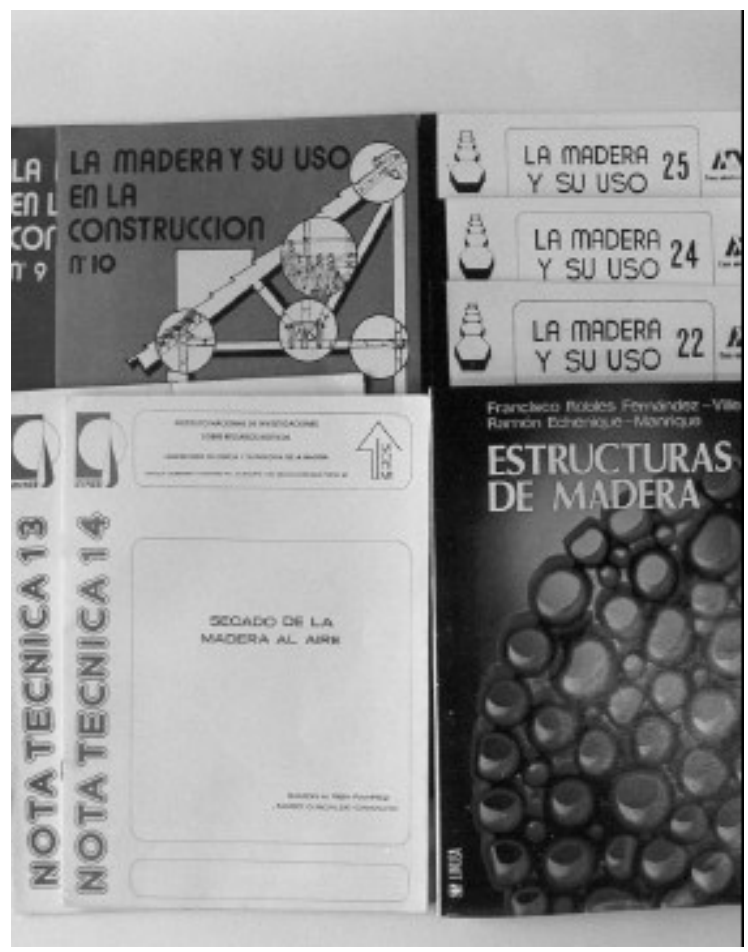

Figura 6. Publicaciones de LACITEMA

Conviene resaltar que a través de los años LACITEMA ha orientado sus esfuerzos tanto al estudio de las especies tropicales como hacia los pinos. Los principales factores que influyeron en la definición del Área de trabajo, han sido la disminución en volumen de madera del trópico a causa de la deforestación, y el auge en el uso de las coníferas en las ciudades de rápido crecimiento.

Para subsanar en parte estos problemas, los estudios de caracterización tecnológica (anatomía, preservación, biodeterioro, propiedades físico-mecánicas) han tenido entre sus metas la de hacer agrupaciones de especies por usos. 


\section{IMPACTO DE LA GLOBALIZACIÓN SOBRE EL FUTURO DE LOS BOSQUES.}

La actual globalización de los mercados impacta las líneas de investigación se deben acabar las líneas de investigación forestal en México. Por un lado, la reciente apertura comercial obliga a los productores forestales a competir con las importaciones de madera, comúnmente de especies coníferas de Norte América y de especies tropicales Asia Sudamérica, cuya oferta supera la demanda mundial actual. El bajo precio al que inicialmente fue introducida esta materia prima importada provocó una crisis en las comunidades forestales, que generalmente aprovechaban y comercializaban la madera en forma de tablas y tablones, así como en la planta industrial, amenazando de nuevo los esfuerzos para conservar el recurso forestal a través de su manejo sostenido.

En este contexto, los profesionales y técnicos forestales experimentados en el trópico insisten en que dentro de la capacitación e investigación se deben abarcar las líneas de "productos forestales" y "plantaciones" en esquemas más diversificado. La importancia de la transformación de la madera, la comercialización, la biodegradación y el abasto de productos forestales se perfilan como temas de investigación prioritarios en los bosques de latifoliadas, en vista de que las importaciones de madera tienden a incrementarse, según información reciente del Banco de México (Gutiérrez, 1944). Asimismo, la experiencias en países asiáticos con comunidades humanas han demostrado:

1) que la conservación y desarrollo del bosque no puede tener lugar sin la participación social; 2) que cualquier actividad de desarrollo debe considerar factores socio-culturales como son la percepción que la población tiene de los productos y beneficios de los árboles, arbustos y hierbas; las especies que pueden satisfacer sus necesidades en forma óptima; cuáles son los factores socio-culturales que han contribuido a la destrucción de los bosques y los cambios de las actitudes que han dado como resultado un desarrollo mal concebido, crecimiento de la población, crisis de las relaciones sociales, etc. (Burch y Parker, 1992).

Por otra parte, la restauración ecológica de áreas degradadas y deforestadas es una necesidad fundamental para asegurar un futuro promisorio para el recurso y los habitantes que dependen de él. Desde sus inicios, el enriquecimiento biológico y las plantaciones han sido enfocadas hacia un número reducido de especies útiles, sin contemplar la biodiversidad que ahora reclama la sociedad.

No obstante, los viveros tiene un lugar de importancia en la tarea de restaurar el medio ambiente y en apoyar los ingresos de la población marginada $y$, debería contar con plántulas necesarias para cubrir la diversidad de usos solicitados por la sociedad moderna. Las pocas especies en los viveros actuales son un reflejo de la poca imaginación apreciación y entendimiento en la reforestación tradicional.

Las plantaciones han formado parte de una política preocupada por cumplir con metas numéricas, sin considerar de manera práctica ni las necesidades, ni las opiniones de base een las comunidades (Dourojeanni, 1985). Desde las plantaciones comerciales, las plantaciones para la protección de cuencas hidrográficas y hasta la deforestación para restablecer el . "Hábitat" de la fauna silvestre, la participación de los centros de investigación ha sido más crítica que participativa. La propagación y transformación y comercialización de un elevado número de especies potencialmente útiles para fines mono-específicos como artesanías, conservación, artículos decorativos, extractivos, fibras, forrajes, medicinales, ornamentales, parques, insumos industriales, madera para construcción, y pulpa y papel, no se daría sin un planteamiento más integral, Además, es urgente desarrollar técnicas más avanzadas para la propagación de especies en peligro de extinción. 


\section{INFLUENCIA DE LOS PROFESIONALES FORESTALES}

Las prácticas tradicionales de la industria forestal y la forma en que los técnicos forestales ejercen la profesión, no se aislan de los cambios. Se ha terminado, casi por completo, la época de las grandes industrias forestales cercanas a los pinares en Durango y Chihuahua. En su lugar, los campesinos han heredado los derechos de esos recursos naturales y toman el mando para conservarlos. Los pequeños productores de madera en México ya se agrupan en, sociedades de producción forestal". La Secretaría de Agricultura y Recursos Hidráulico (SARH) ha descentralizado sus facultades de otorgar permisos y ha eliminado la asesoría en forma definitiva. Los profesionales que ahora ofrecen apoyo para asesorar a los grupos de campesinos con vocación forestal, conocidos como, sociedades de producción forestal" han formado asociaciones civiles que proporcionan servicios técnicos en manejo $y$ comercialización de los bosques y selvas. Comúnmente, en estas asociaciones civiles se integran profesionistas multidisciplinarios, constituidas por forestales y biólogos, principalmente. Estas son una especie de "bufetes" para encargarse de forma inicial del ordenamiento del recurso forestal, por medio de los llamados Estudios de Manejo Integral (EMI) que son a la vez requisitos para el otorgamiento de los permisos de aprovechamiento de la SARH (Arguelles, 1991 ).

En vista de que el estado eliminó la asistencia técnica gratuita al productor, se ha incrementado notoriamente la oferta de los servicios técnicos proporcionados por estas asociaciones civiles. El trabajo que desempeñan se realiza en conjunto con las asociaciones campesinas en todas las fases del aprovechamiento de sus bosques, aún cuando la labor inicial es la de preparar el EMI.

Para proporcionar los servicios técnicos como son : conseguir los permisos, capacitación de productores y comercialización, estas asociaciones civiles comúnmente financian sus labores de dos formas: 1) cobran a los campesinos una cuota por cada metro cúbico de madera y, 2) consiguen fondo para sus proyectos productivos en instituciones estatales y/o los dedicadas a la conservación .

Sin embargo el hecho de tener que cobrar una parte sus salarios en función del volumen de producción y muchas veces a costa de los grupos (y bosques) marginados, se presta en ocasiones para el sobreaprovechamiento de los recursos forestales y debilita el panorama para la conservación. Aun en donde hay perspectivas para el manejo sostenido, los costos para mantener los servicios técnicos elevan los de producción y reducen la competitividad de la pequeña empresa ejidal (Argüelles, op. cit.). Lo anterior abre una ventana especial para las sociedades civiles que sean capaces de conseguir recursos, promover actividades como la silvicultura y apoyar en la comercialización de los recursos. Hasta la fecha, ninguna de las asociaciones civiles en México cuenta con infraestructura propia para apoyar los esfuerzos de los ejidos en líneas de productos forestales o viveros.

Esta situación obliga a un replanteamiento en el aprovechamiento del bosque y por ende, de las actividades de LACITEMA. Para promover la sostenibilidad del bosque es obvia la necesidad de reconciliar la ecología y los intereses de la sociedad moderna en un sentido amplio. Hasta la fecha, el Instituto de Ecología, A. C., ha estado activo en la investigación científica sobre temas esenciales, a pesar de no tener una unidad de investigación dedicada específicamente para los fines del desarrollo rural.

Sin embargo, se nota claramente el esfuerzo de integrar grupos de trabajo en proyectos para el sector social en los ordenamientos territoriales y en el trabajo realizado en las reservas de la Biosfera. 


\section{ORIENTACIÓN DEL NUEVO DEPARTAMENTO}

En el futuro las líneas de investigación del antiguo LACITEMA se orientarán hacia los retos contemporáneos de la conservación de los bosques a través de su manejo y aprovechamiento sostenido. Se busca ampliar el enfoque de uso de la madera aserrada hacia la utilización de tecnologías adecuadas para agregar mayor valor a la materia de los bosques mexicanos, sin perder de vista la apertura comercial. Se pretende enfocar la experiencia de los recursos humanos existen en labores académicas y productivas en el ámbito del bosque y las empresas campesinas o industriales que dependen de éste. Por otra parte se contempla la necesidad de formar otro grupo de trabajo en manejo y conservación de los recursos forestales. En el área de conservación del bosque, las líneas de investigación se concentrarán en manejo integral de los recursos provenientes del bosque. Por ello, la producción de plantas para su incorporación en los sistemas productivos (agroforestal y silviculturales) y los enlaces con otros grupos disciplinarios ya consolidados en el IE se perfila como una manera de integrar investigación ecológica y sus esfuerzos en la restauración de bosque.

Inicialmente se prevé la necesidad de reorientar y/o substituir algunos de los recursos humanos del LACITEMA. Un requisito adicional es contratar un número mínimo de investigadores ya experimentados en la investigación y desarrollo forestal. De otra forma se corre el riesgo de quedar corto en concretar una masa crítica para los fines anteriormente concretados.

El trabajo en proyectos, tanto académicos como de desarrollo, se deberá realizar en forma conjunta y organizada sobre unas pocas áreas que tengan un potencial para el desarrollo adecuado del complejo bosque-comunidad-industria.

El Departamento de Productos Forestales y Conservación de Bosques del
Instituto de Ecología, A.C., estará formado por el vínculo entre los investigadores del instituto, principalmente investigadores de los Departamentos de Ecología Vegetal, Flora de Veracruz, Diagnóstico Regional y Ecosistemas Templados, con el personal de investigación del LACITEMA. La investigación que realice el Departamento constituirá una nueva dimensión de tecnología y ecología aplicada en los ecosistemas forestales en México.

\section{Objetivos}

Los objetivos principales del nuevo departamento son los siguientes:

1) Efectuar investigación científica, de ingeniería y tecnología para buscar el aprovechamiento sostenido de los recursos forestales, particularmente, la madera.

2) Realizar investigación y evaluación acerca de los sistemas silvícolas y agrosilvícolas, así como de un impacto ecológico.

3) Hacer estudios sobre retauración ecológica en áreas desprovistas de su vegetación natural $y / 0$ fragmentadas a través de la Unidad de Propagación .

4) Diseñar planes de manejo y de utilización de los recursos forestales, considerando la participación social.

5) Facilitar la comunicación entre los investigadores, usuarios y productores del recurso forestal mediante el fortalecimiento de la revista editada por el departamento que de ahora en adelante se denominará "Madera y Bosques".

6) A través de la Unidad de Información, proporcionar información, capacitación y asistencia técnica a la industria de aprovechamiento de recursos forestales y a las comunidades humanas que lo demanden. 
Para cumplir con el primero de estos objetivos, se llevarán a cabo los estudios básicos y de frontera que el desarrollo del país exige, buscando siempre generar los avances tecnológicos más necesarios para el apoyo del sector industrial, en las áreas de competencia del Departamento, de acuerdo con las líneas de investigación seleccionadas. Las áreas de estudio relacionadas con este objetivo se integrarán en un sub-departamento llamado productos forestales.

Las áreas de estudio relacionadas con los objetivos 2 a 4, estarán aglobadas dentro del sub-departamento de manejo y conservación. Para el segundo de los objetivos, en los sitios elegidos para la investigación de los sistemas de producción, se realizarán actividades de conservación de manera práctica como parte de la asesoría en servicios técnicos. Esto, con el fin de asegurar la validación de las prácticas culturales y técnicas propuestas. El financiamiento de las técnicas agrosilvícolas y la adecuada transformación de la materia prima en productos forestales, se efectuará mediante proyectos académicos y productivos, con la participación del sector privado y público. Para complementar la investigación sobre sistemas de producción sostenida, se integrarán grupos de trabajo interdisciplinario para validar la investigación.

El enfoque del grupo se centra en ecosistemas y agroecosistemas provenientes del Bosque Mesófilo de Montaña; mismo que presenta la oportunidad de cubrir un amplio rango de investigación desde la autoecología y silvicultura, hasta los aspectos socioeconómicos y la comercialización de sus productos forestales. Por otra parte, el Departamento actualmente tiene una investigación en agroecosistemas y selvas bajas caducifolia en los estados de Guerrero y Jalisco, así como en ecosistemas de la Península de Yucatán.

Con relación al tercer objetivo, el de enfrentarse a los retos de restauración ecológica en áreas desprovistas de su vegetación natural y/o fragmentadas, se buscará apoyo para crear la Unidad de
Propagación. En la Unidad de Propagación se pretende integrar tecnología de punta con prácticas hortícolas en el Jardín Botánico, Francisco J. Clavijero, para la conservación in situ y ex situ de especies amenazadas y en peligro de extinción y de otra índole de importancia antropogénica. En la Unidad de Propagación se realizará propagación vegetativa, complementada con el aislamiento de razas prometedoras de endo-micorrizas. El inóculo del germoplasma produciría plántulas para la restauración ecológica en ambientes muy variados que incluyen plántulas para la reforestación mecanizada.

El cuarto objetivo guarda cierta relación con el segundo debido a que la agrosilvicultura en un contexto más amplio de estrategias de desarrollo con base en la relación árbol-personas, contempla estudios de manejo integral, la incorporación de la mujer en proyectos de desarrollo silvícola, la organización de las comunidades humanas para la producción y la comercialización de los productos maderables y no maderables.

En cuanto al quinto objetivo, se ratifica la intención de fortalecer la comunicación mediante la reestructuración de la revista "Madera y su Uso", y el Boletín LACITEMA. Con el fin de ampliar su alcance y captar el interés de un mayor número de lectores, se han reestructurado su Comité Editorial, formato, contenido y periodicidad. Se ha cambiado de nombre de la revista al de "Madera y Bosques" y se fusiona ésta con el Boletín. La revista inicia con el Volumen 1, Número 1.

Finalmente el sexto objetivo, se fundamenta tanto en la necesidad de fortalecer y consolidar la Unidad de Información, como la de contar con un centro especializado en asesoría técnica, certificación de calidad y desarrollo de productos en el área del aprovechamiento de los recursos forestales, el cual actualmente no existe en el país y tiene una amplia demanda por la industria nacional. Los especialistas del Departamento están singularmente calificados para prestar esta asesoría a los industriales y ya se están tomando los primeros pasos para lograr la 
acreditación del laboratorio ante los organismos correspondientes. Además de ser consistentes con la filosofía de las autoridades nacionales en el sistema de investigación científica y tecnológica, de enlazar a los centros de investigación con la industria, esta será una de las fuentes principales de recursos externos para el Departamento; esto sin dejar de lado la atención de las necesidades de información que también tienen otros usuarios de la madera, como son estudiantes, productores, organismos gubernamentales y no gubernamentales, etc., contando con la estructura de la Unidad de Información como respaldo.

\section{LINEAS GENERALES DE INVESTIGACIÓN}

Los trabajos fundamentales en el área de productos forestales se centrarán en muchas de las actividades tradicionales del Departamento, como son: Caracterización de especies mexicanas poco conocidas a con potencial comercial; secado, anatomía, propiedades físico-mecánicas y acabados. También se continuarán los trabajos sobre usos estructurales de diversos productos: Madera aserrada, madera reconstituida, tableros, maderas laminada, uniones, conectores y adhesivos. Otra área de interés para el Departamento la constituirán, como de costumbre, la elaboración de normas técnicas, reglamentos y manuales.

Entre las actividades que se pueden considerar novedosas para este grupo están la química de la madera y silvoquímica, y los estudios tradicionales sobre tratabilidad, preservación y biodeterioro

La nueva área que se incluirá en las actividades del Departamento es la de manejo y conservación, en la cual se le dará énfasis a trabajos sobre manejo y utilización de recursos, particularmente de sistemas de producción agrosilvícolas y sistemas agrosilvopastoriles.

Asimismo, se realizarán estudios sobre manejo y utilización de recursos forestales, sin dejar de considerar la participación de la mujer en proyectos agrosilvícolas, la organización campesina para la producción, estudios de socio-economía, comercialización y aprovechamientos.

Se pretende también incursionar en el área de la ecología forestal con trabajos sobre silvicultura de especies mexicanas, ecología forestal (fotosíntesis y productividad neta primaria ) y ecofisiología.

En las áreas de restauración ecológica, se harán trabajos sobre propagación y enriquecimiento biológico.

Finalmente, no se descuidará el área de proyectos de desarrollo y productivos, que tendrá como metas la acreditación de un laboratorio de certificación de la calidad para productos forestales. Se seguirá ofreciendo asesoría sobre ingeniería y tecnología de productos forestales, adecuación y reconversión de industrias, así como servicios técnicos para estudios de manejo integral.

\section{INFRAESTRUCTURA}

La infraestructura del Departamento, del Jardín Botánico, del Centro de Investigaciones Costeras del a Mancha, las Reservas de la Biosfera, laboratorios en la sede de Xalapa y la Unidad de información, constituyen una base sólida para investigadores y técnico del Departamento. Se espera aumentar la capacidad para la realización de investigaciones y la consecución de proyectos productivos, contribuyendo así de manera teórica y práctica con el que hacer en la ciencia forestal y de los productores forestales en beneficio de los bosques y selvas de México.

\section{RECONOCIMIENTOS}

Agradecemos la valiosa cooperación de Guadalupe Bárcenas P. Por haber hecho la primera revisión del documento; Laura C. Ruelas M. Por la aportación de ideas en el ámbito de los estudios socioeconómicos y quien tuvo a su cargo la edición final; así como a Enrique Martínez Pinillos por las fotografías presentadas en este trabajo. 


\section{REFERENCIAS}

Argüelles, L.A. 1991. Experiencias en desarrollo rural: el caso del Plan Piloto Forestal de Quintana Roo, México. In: P.V. Johnson, Ed Development Strategies and Natural Resource Management. Proceedings of the Humid Lowlands Conference.

Bárcenas P., G. M., R. Romero Rejón y R. Echenique-Manrique.1980. Especies maderables de la Selva Lacandona. La madera y su Uso en la Construcción No. 6 INIREBLACITEMA, Xalapa, Ver.

Bárcenas P., G. M. 1994 Caracterización tecnológica de veinte especies maderables de la Selva Lacandona. Madera y Bosques 1 ( 1 ) Instituto de Ecología, A. C., Xalapa, Ver. (en prensa )

Burch Jr., W. R. y J.K. Parker, Eds. 1992. Social science applications in asian agroforestry. Winrock International and Oxford \& IBT Publishing, EUA.

Dávalos S., R., F.F. Wangaard y R. Echenique-Manrique. 1977. Clasificación de la madera de pinos mexicanos. La madera y su Uso en la Construcción No. 2. INIREBLACITEMA, Xalapa, Ver.

Dávalos S., R.1994. Ayudas de diseño. In: M. Elorza, ed. Manual de construcción de Estructuras Ligeras de Madera ( COFAN ). México, D.F. (en prensa).

Dávalos S., R., V. R. Ordóñez C., R. Echenique-Manrique y $\mathrm{F}$. Robles FV. 1984 Stress grading of mexican pine lumber. Memorias de la Reunión del Grupo de Ingeniería de la Madera de IUFRO, Xalapa, Ver.

Departamento del Distrito Federal. 1987 a. Reglamento de Construcciones para el Distrito Federal. 10ma. ed Serie
Leyes y Códigos de México. Porrúa, México, D. F.

Departamento del Distrito Federal. 1987 b. Normas Técnicas Complementarias para el Diseño y Construcción de Estructuras de Madera. Gaceta Oficial del Departamento Federal Quinta Epoca No. 48. México, D. F. 1 de Diciembre de 1987.

Dirección General de Normas. 1983. NMXC-178-1983: Preservadores solubles en agua y aceite. México, D. F.

Dirección General de Normas. 1983. NMXC-222-1983: Prevención al ataque por termitas. México, D. F.

Dirección General de Normas. NMX-C-2241983: Dimensiones de la madera aserrada para su uso en le construcción. México, D. F.

Dirección General de Normas. 1985. NMX-C239-1985: Calificación y clasificación visual para la madera de pino en usos estructurales. México, D. F.

Dirección General de Normas. 1994. NMXC-1994: Especificaciones de comportamiento para tableros a base de madera para su uso en viviendas. (en trámite de aprobación). México, D. F.

Dourojeanni, M.J. 1985. Los retos de la educación forestal al filo del siglo $X X I$. IX Congreso Forestal Mundial, México, D. F. (mimegrafiado)

Echenique-Manrique, R., R. Dávalos S. y F. Robles F-V. 1987. Informe final al CIID. Calificación y clasificación visual para la madera de pino en usos estructurales ("Timber grading México"). Fase III. INREB-LACITEMA, Xalapa,Ver.

Erdoiza S., J. y M.I. Castillo M. 1989. Susceptibilidad de impregnación con preservadores de cincuenta especies 
maderables mexicanas. La madera y su Uso No. 22, Instituto de Ecología, A. C., Xalapa, Ver.

Evans., P. A. y M. A. Arizmendi. 1991. International directory of forestry \& forest products libraries. Forestry Library, University of California, Berkeley, C. A.

Gutiérrez, E. 1994. Mayor importación de bienes de consumo, primera señal del TLC en el comercio exterior. El Financiero, viernes 22 de abril de 1994.

Laboratorio de Ciencia y Tecnología de la Madera (INIREB). Catálogo de productos TYPAM: INIREBLACITEMA, Xalapa, Ver.

Martínez-Pinillos C., E. 1984. Secadora solar para madera. Nota Técnica No. 10, INIREB-LACITEMA, Xalapa, Ver.

Martínez-Pinillos C., E. 1990. Laminadodoblado de la madera. La madera y su Uso No. 24, Instituto de Ecología, A. C., Xalapa, Ver.

Ordóñez C., V. R. y R. Dávalos S. 1985. Manual de clasificación visual para madera estructural de pino. Nota Técnica No. 12, INIREB-LACITEMA, Xalapa, Ver.

Ordóñez C., V.R., G.M. Bárcenas P. y A. Quiroz S. 1989. Características físico-mecánicas de la madera de diez especies de San Pablo Macuiltianguis, Oaxaca. La madera y su Uso No. 21, Instituto de Ecología, A.C., Xalapa, Ver.

Ortega E., F., L. Guerrero O., T.F. Carmona V. y C. Córdova N. 1988. Anatomía de la madera de veintiocho especies de Cosautlán de Carvajal, Veracruz. Serie "Angiospermas arbóreas de México, No. 1". La madera y su Uso No. 19, INIREB-LACITEMA,
Xalapa, Ver.

Pérez M., V., G. Heras S. y R. EcheniqueManrique. 1977. Riesgo a la pudrición de la madera en diferentes climas de México. La Madera y su Uso en la Construcción No.1, INIREBLACITEMA, Xalapa, Ver.

Pérez M., V., G.M. Bárcenas P. y R. Echenique-Manrique. 1981. Prevención y control de daño por termitas en estructuras con madera. La madera y su Uso en la Construcción No. 7, INIREBLACITEMA, Xalapa, Ver.

Ricalde C., M.O. 1988. Manual para diseño de estructuras de madera. Cap. 1.1: La madera en México. Instituto de Ecología, A.C. Xalapa, Ver.

Robles F-V., F., R. Dávalos S. y M.O. Ricalde C. 1988. Comentarios a las normas técnicas complementarias para diseño y construcción de estructuras de madera versión 1987. La Madera y su Uso No. 15, INIREB-LACITEMA, Xalapa, Ver.

Ruelas M., L.C. 1993. Boletín LACITEMA No. 48. Instituto de Ecología, A.C. Xalapa, Ver. 